\title{
New Set-Valued Integral in a Banach Space
}

\author{
Cai-Li Zhou' ${ }^{1}$ and Fu-Gui Shi \\ ${ }^{1}$ College of Mathematics and Information Science, Hebei University, Baoding 071002, China \\ ${ }^{2}$ School of Mathematics and Statistics, Beijing Institute of Technology, Beijing 100081, China
}

Correspondence should be addressed to Cai-Li Zhou; hyyanyiyi@163.com

Received 26 October 2014; Accepted 27 February 2015

Academic Editor: Wilfredo Urbina

Copyright ( 2015 C.-L. Zhou and F.-G. Shi. This is an open access article distributed under the Creative Commons Attribution License, which permits unrestricted use, distribution, and reproduction in any medium, provided the original work is properly cited.

We introduce and study a new set-valued integral of scalar-valued functions with respect to a set-valued measure in a Banach space. We investigate some properties and convergence theorems for this kind of integral.

\section{Introduction}

The need for set-valued measures first arose in mathematical economics when Vind [1] studied equilibrium theory for exchange economies with production. Since then the subject of set-valued measures has received a lot of attention and, as it turned out, developed to be the set-valued analogue of the classical theory of vector measures (see, e.g., [2-9]). Accordingly, various developments in mathematical economics and optimal control have led to the study of integrals with respect to set-valued measures and many papers dealt with the basic theory of these integrals. Among them, as an extension of the integral of scalar-valued functions with respect to vector measures, Papageorgiou [5] first introduced the integral of scalar-valued functions with respect to a set-valued measure by the bilinear integral of Dinculeanu [10]. Since then, Kandilakis [11] introduced an integral of bounded real-valued functions with respect to a set-valued measure using the set of Kluvánek-Knowles type integrals [12]. Wu et al. [13] introduced the set-valued Bartle integral which is the set of BartleDunford-Schwartz type integrals [14]. The set-valued Bartle integral has also been deeply considered by Zhang et al. [9]. Precupanu and Croitoru $[15,16]$ introduced a multivalued Gould type integral for a real-valued function with respect to a set-valued measure. Using [15, 16], Gavriluț $[17,18]$ introduced a Gould type integral of a real-valued function with respect to a multisubmeasure. Recently, Precupanu and Satco [19] introduced an Aumann-Gould integral in which the selectors are Gould integrals of real-valued functions with respect to vector-valued measures [20]. Precupanu et al. [21] introduced a set-valued Gould type integral of a real function with respect to a set-valued fuzzy measure.

In the present paper, we introduce and study a new setvalued integral of scalar-valued functions with respect to a set-valued measure. Unlike various ways of defining such an integral (e.g., Papageorgiou way, Kandilakis way, and $\mathrm{Wu}$, Zhang, and Wang way), our integral does not depend on measure selections of the given set-valued measure and is a Pettis type set-valued integral of scalar-valued functions with respect to a set-valued measure. The result is an extension of the Kluvánek-Lewis type integral [22-24], which is a Pettis type weak integral of scalar-valued functions with respect to a vector-valued measure. We investigate some properties and establish convergence theorems for this kind of integral. Finally, we discuss the relationship between this new integral and the set-valued Bartle integral.

The paper is structured as follows. In Section 2, we state some basic concepts and preliminary results which will be used in the sequel. In Section 3, we first introduce the new integral with some natural properties. And then, we prove Vitali type convergence theorem and dominated convergence theorem for this kind of integral. Finally, it is shown that, under some natural assumptions on the Banach space and on the set-valued measure, our integral is deduced to the setvalued Bartle integral, which shows that our integral is also an extension of the set-valued Bartle integral. 


\section{Preliminaries}

Throughout this paper, let $(\Omega, \mathscr{A}, \mu)$ be a complete finite measure space where $\Omega$ is a nonempty set, $\mathscr{A}$ is a $\sigma$-algebra of subsets of $\Omega$, and $\mu$ is a measure. Let $(\mathscr{X},\|\cdot\|)$ be a real separable Banach space with its dual space $\mathscr{X}^{*}$. Let

$$
\begin{aligned}
& \mathscr{P}_{0}(\mathscr{X})=\{A \subset \mathscr{X}: A \text { is a nonempty subset of } X\}, \\
& \mathscr{P}_{b(f)(c)}(\mathscr{X}) \\
& \quad=\left\{A \in \mathscr{P}_{0}(\mathscr{X}): A \text { is bounded (closed) (convex) }\right\}, \\
& \mathscr{P}_{\text {wkc }}(\mathscr{X}) \\
& \quad=\left\{A \in \mathscr{P}_{0}(\mathscr{X}): A \text { is weakly compact and convex }\right\} .
\end{aligned}
$$

For $A, B \in \mathscr{P}_{f}(\mathscr{X})$, the Hausdorff metric $H$ of $A$ and $B$ is defined by

$$
H(A, B)=\max \left\{\sup _{a \in A} d(a, B), \sup _{b \in B} d(b, A)\right\},
$$

where $d(a, B)=\inf _{b \in B}\|a-b\|$. For $A \subset \mathscr{X}$, the number $|A|$ is defined by $|A|=H(A,\{0\})=\sup _{x \in A}\|x\|$. Note that $\left(\mathscr{P}_{\text {wkc }}(\mathscr{X}), H\right)$ is a complete metric space.

Throughout this work we will denote by $\sigma(\cdot, A)$ the support function of the set $A \subset \mathscr{X}$ defined by

$$
\sigma\left(x^{*}, A\right)=\sup _{x \in A} x^{*}(x), \quad x^{*} \in \mathscr{X}^{*} .
$$

The support function satisfies the properties $\sigma\left(x^{*}, A+B\right)=$ $\sigma\left(x^{*}, A\right)+\sigma\left(x^{*}, B\right)$ and $\sigma\left(x^{*}, \lambda A\right)=\lambda \sigma\left(x^{*}, A\right)$ for all $A, B \in$ $\mathscr{P}_{0}(\mathscr{X}), x^{*} \in \mathscr{X}^{*}$ and $\lambda \geq 0$. In particular,

$$
H(A, B)=\sup _{\left\|x^{*}\right\| \leq 1}\left|\sigma\left(x^{*}, A\right)-\sigma\left(x^{*}, B\right)\right|
$$

whenever $A, B$ are two convex sets.

Definition 1 (see [6]). Let $(\Omega, \mathscr{A})$ be a measurable space. A set-valued set function $\mathscr{M}: \mathscr{A} \rightarrow \mathscr{P}_{0}(\mathscr{X})$ is said to be a setvalued measure if it satisfies the following two conditions:

(1) $\mathscr{M}(\emptyset)=\{0\}$;

(2) if $A_{1}, A_{2}, \ldots$ are in $\mathscr{A}$, with $A_{i} \cap A_{j}=\emptyset$ for $i \neq j$, then

$$
\mathscr{M}\left(\bigcup_{n=1}^{\infty} A_{n}\right)=\sum_{n=1}^{\infty} \mathscr{M}\left(A_{n}\right)
$$

where $\sum_{n=1}^{\infty} \mathscr{M}\left(A_{n}\right)=\left\{x \in \mathscr{X}: x=\sum_{n=1}^{\infty} x_{n}\right.$ (unc.conv.), $x_{n} \in$ $\left.\mathscr{M}\left(A_{n}\right), n \geq 1\right\}$.

In particular, $\mathscr{M}: \mathscr{A} \rightarrow \mathscr{P}_{\text {wkc }}(\mathscr{X})$ is a set-valued measure if and only if $\sigma\left(x^{*}, \mathscr{M}(\cdot)\right)$ is a scalar-valued measure for all $x^{*} \in \mathscr{X}^{*}$ (cf. [6]).

A vector-valued measure $m: \mathscr{A} \rightarrow \mathscr{X}$ satisfying $m(A) \epsilon$ $\mathscr{M}(A)$ for all $A \in \mathscr{A}$ is said to be a selection of the set-valued measure $\mathscr{M}$. We denote the set of all selections of $\mathscr{M}$ by $S_{\mathscr{M}}$.
For a set-valued measure $\mathscr{M}$, we have the notion of total variation $|\mathscr{M}|$ defined by $|\mathscr{M}|(A)=\sup \sum_{i=1}^{n}\left|\mathscr{M}\left(A_{i}\right)\right|$ for $A \epsilon$ $\mathscr{A}$, where the supremum is taken over all finite measurable partitions $\left\{A_{1}, \ldots, A_{n}\right\}$ of $A$. It is easy to check that $|\mathscr{M}|$ is a positive measure. If $|\mathscr{M}|(\Omega)<\infty$, then we say that $\mathscr{M}$ is of bounded variation. In this case the sums in the definition of $\sum_{n=1}^{\infty} \mathscr{M}\left(A_{n}\right)$ are absolutely convergent. We call that $\mathscr{M}$ is $\mu$ continuous, where $\mu$ is a single-valued vector measure; if, for any $A \in \mathscr{A}, \mu(A)=0$, then $\mathscr{M}(A)=\{0\}$.

A Banach space $\mathscr{X}$ has the Radon-Nikodým property (RNP) if, for each finite measure space $(\Omega, \mathscr{A}, \mu)$ and each $\mu$-continuous $\mathscr{X}$-valued measure $m: \mathscr{A} \rightarrow \mathscr{X}$ of bounded variation, there exists a Bochner integrable function $f: \Omega \rightarrow$ $\mathscr{X}$ such that $m(A)=\int_{A} f(\omega) \mathrm{d} \mu(\omega)$ for all $A \in \mathscr{A}$. A measurable set-valued function $X: \Omega \rightarrow \mathscr{P}_{0}(\mathscr{X})$ is said to be a Radon-Nikodým derivative of the set-valued measure $\mathscr{M}$ with respect to $\mu$ if $\mathscr{M}(A)=\int_{A} X(\omega) \mathrm{d} \mu(\omega)$ for all $A \in \mathscr{A}$ and we write $\mathrm{d} \mathscr{M}=X \mathrm{~d} \mu$.

Definition 2 (see [24]). Let $m: \mathscr{A} \rightarrow \mathscr{X}$ be a vector-valued measure and let $f: \Omega \rightarrow \mathbb{R}$ be $\mathscr{A}$-measurable. $f$ is said to be Kluvánek-Lewis integrable with respect to $m$ (for short, (KL) $m$-integrable) if

(1) $f$ is $x^{*} m$-integrable for each $x^{*} \in \mathscr{X}^{*}$;

(2) for each $A \in \mathscr{A}$, there exists a vector $x_{A} \in \mathscr{X}$ such that

$$
x^{*}\left(x_{A}\right)=\int_{A} f(\omega) \mathrm{d} x^{*} m(\omega)
$$

for each $x^{*} \in \mathscr{X}^{*}$. In that case, we define (KL) $\int_{A} f(\omega) \mathrm{d} m(\omega)=x_{A}$ for each $A \in \mathscr{A}$.

Note that by the Hahn-Banach theorem we know that the integral is well defined and unique.

\section{Main Results}

In the sequel, let $L^{1}(\Omega, \mathbb{R},|\mathscr{M}|)$ be the space of all functions $f: \Omega \rightarrow \mathbb{R}$ which are $\mathscr{A}$-measurable and $|\mathscr{M}|$-integrable. We first introduce the new integral as follows.

Definition 3. Let $\mathscr{M}: \mathscr{A} \rightarrow \mathscr{P}_{\text {wkc }}(\mathscr{X})$ be a set-valued measure and $f: \Omega \rightarrow \mathbb{R}$ an element of $L^{1}(\Omega, \mathbb{R},|\mathscr{M}|) . f$ is said to be Kluvánek-Lewis integrable with respect to $\mathscr{M}$ (for short, (KL) $\mathscr{M}$-integrable) if

(1) $f$ is $\sigma\left(x^{*}, \mathscr{M}(\cdot)\right)$-integrable for each $x^{*} \in \mathscr{X}^{*}$;

(2) for each $A \in \mathscr{A}$, there exists a $W_{A} \in \mathscr{P}_{\text {wkc }}(\mathscr{X})$ such that

$$
\sigma\left(x^{*}, W_{A}\right)=\int_{A} f(\omega) \mathrm{d} \sigma\left(x^{*}, \mathscr{M}(\omega)\right)
$$

for each $x^{*} \in \mathscr{X}^{*}$. In the case, we write

$$
W_{A}=(\mathrm{KL}) \int_{A} f(\omega) \mathrm{d} \mathscr{M}(\omega)
$$

for each $A \in \mathscr{A}$ and call it set-valued Kluvánek-Lewis integral of $f$ with respect to $\mathscr{M}$ on $A$. 
Remark 4. The set-valued Kluvánek-Lewis integral is a generalization of the Kluvánek-Lewis integral. Note that when the set-valued measure $\mathscr{M}$ is degenerated into a vector-valued measure $m$, the set $W_{A}$ is reduced to a vector in $\mathscr{X}$ and the equality $\sigma\left(x^{*}, W_{A}\right)=\int_{A}^{A} f(\omega) \mathrm{d} \sigma\left(x^{*}, \mathscr{M}(\omega)\right)$ turns into

$$
x^{*}\left(W_{A}\right)=\int_{A} f(\omega) \mathrm{d} x^{*} m(\omega)
$$

for each $x^{*} \in \mathscr{X}^{*}$.

Example 5. Let $\mathscr{M}: \mathscr{A} \rightarrow \mathscr{P}_{\text {wkc }}(\mathscr{X})$ be a set-valued measure. If $f=\sum_{i=1}^{n} \lambda_{i} \chi_{A_{i}}$ is a nonnegative simple function, then $f$ is (KL) $\mathscr{M}$-integrable and

$$
(\mathrm{KL}) \int_{A} f(\omega) \mathrm{d} \mathscr{M}(\omega)=\sum_{i=1}^{n} \lambda_{i} \mathscr{M}\left(A_{i} \cap A\right)
$$

for each $A \in \mathscr{A}$.

Proof. Obviously, $f=\sum_{i=1}^{n} \lambda_{i} \chi_{A_{i}}$ is $\sigma\left(x^{*}, \mathscr{M}(\cdot)\right)$-integrable for each $x^{*} \in \mathscr{X}^{*}$ and

$$
\begin{aligned}
& \int_{A} \sum_{i=1}^{n} \lambda_{i} \chi_{A_{i}}(\omega) \mathrm{d} \sigma\left(x^{*}, \mathscr{M}(\omega)\right) \\
& =\sum_{i=1}^{n} \lambda_{i} \int_{A} \chi_{A_{i}}(\omega) \mathrm{d} \sigma\left(x^{*}, \mathscr{M}(\omega)\right) \\
& =\sum_{i=1}^{n} \lambda_{i} \sigma\left(x^{*}, \mathscr{M}\left(A_{i} \cap A\right)\right)
\end{aligned}
$$

for each $A \in \mathscr{A}$. On the other hand, by the properties of support functions, we have

$$
\sigma\left(x^{*}, \sum_{i=1}^{n} \lambda_{i} \mathscr{M}\left(A_{i} \cap A\right)\right)=\sum_{i=1}^{n} \lambda_{i} \sigma\left(x^{*}, \mathscr{M}\left(A_{i} \cap A\right)\right)
$$

for each $x^{*} \in \mathscr{X}^{*}$. It follows from the above two equalities that

$$
\sigma\left(x^{*}, \sum_{i=1}^{n} \lambda_{i} \mathscr{M}\left(A_{i} \cap A\right)\right)=\int_{A} \sum_{i=1}^{n} \lambda_{i} \chi_{A_{i}}(\omega) \mathrm{d} \sigma\left(x^{*}, \mathscr{M}(\omega)\right)
$$

for each $x^{*} \in \mathscr{X}^{*}$. Since $\mathscr{M}\left(A_{i} \cap A\right) \in \mathscr{P}_{\text {wkc }}(\mathscr{X})$, we have $\sum_{i=1}^{n} \lambda_{i} \mathscr{M}\left(A_{i} \cap A\right) \in \mathscr{P}_{\text {wkc }}(\mathscr{X})$. Thus, $f$ is (KL) $\mathscr{M}$-integrable and

$$
(\mathrm{KL}) \int_{A} f(\omega) \mathrm{d} \mathscr{M}(\omega)=\sum_{i=1}^{n} \lambda_{i} \mathscr{M}\left(A_{i} \cap A\right)
$$

This completes the proof.

In what follows, some properties of the set-valued Kluvánek-Lewis integral will be given.
Theorem 6. Let $\mathscr{M}: \mathscr{A} \rightarrow \mathscr{P}_{w k c}(\mathscr{X})$ be a set-valued measure, $f, g: \Omega \rightarrow \mathbb{R}(K L) M$-integrable, and $\lambda \geq 0$. Then

(1) $f+g$ is (KL) $M$-integrable and

$$
\begin{aligned}
& (K L) \int_{A}\{f(\omega)+g(\omega)\} \mathrm{d} \mathscr{M}(\omega) \\
& =(K L) \int_{A} f(\omega) \mathrm{d} \mathscr{M}(\omega)+(K L) \int_{A} g(\omega) \mathrm{d} \mathscr{M}(\omega)
\end{aligned}
$$

for each $A \in \mathscr{A}$;

(2) $\lambda f$ is (KL) $\mathscr{M}$-integrable and

$(K L) \int_{A} \lambda f(\omega) \mathrm{d} \mathscr{M}(\omega)=\lambda(K L) \int_{A} f(\omega) \mathrm{d} \mathscr{M}(\omega)$

for each $A \in \mathscr{A}$.

Proof. (1) Since $f$ and $g$ are (KL) $\mathscr{M}$-integrable, $f$ and $g$ are $\sigma\left(x^{*}, \mathscr{M}(\cdot)\right)$-integrable for each $x^{*} \in \mathscr{X}^{*}$ and there exist

$$
(\mathrm{KL}) \int_{A} f(\omega) \mathrm{d} \mathscr{M}(\omega), \quad(\mathrm{KL}) \int_{A} g(\omega) \mathrm{d} \mathscr{M}(\omega)
$$

in $\mathscr{P}_{\text {wkc }}(\mathscr{X})$ such that

$$
\begin{gathered}
\sigma\left(x^{*},(\mathrm{KL}) \int_{A} f(\omega) \mathrm{d} \mathscr{M}(\omega)\right)=\int_{A} f(\omega) \mathrm{d} \sigma\left(x^{*}, \mathscr{M}(\omega)\right), \\
\sigma\left(x^{*},(\mathrm{KL}) \int_{A} g(\omega) \mathrm{d} \mathscr{M}(\omega)\right)=\int_{A} g(\omega) \mathrm{d} \sigma\left(x^{*}, \mathscr{M}(\omega)\right)
\end{gathered}
$$

for each $A \in \mathscr{A}$ and $x^{*} \in \mathscr{X}^{*}$. Obviously, $f+g$ is $\sigma\left(x^{*}, \mathscr{M}(\cdot)\right)$ integrable and, by properties of support functions, we have

$$
\begin{aligned}
& \sigma\left(x^{*},(\mathrm{KL}) \int_{A} f(\omega) \mathrm{d} \mathscr{M}(\omega)+(\mathrm{KL}) \int_{A} g(\omega) \mathrm{d} \mathscr{M}(\omega)\right) \\
&= \sigma\left(x^{*},(\mathrm{KL}) \int_{A} f(\omega) \mathrm{d} \mathscr{M}(\omega)\right) \\
&+\sigma\left(x^{*},(\mathrm{KL}) \int_{A} g(\omega) \mathrm{d} \mathscr{M}(\omega)\right) \\
&= \int_{A} f(\omega) \mathrm{d} \sigma\left(x^{*}, \mathscr{M}(\omega)\right)+\int_{A} g(\omega) \mathrm{d} \sigma\left(x^{*}, \mathscr{M}(\omega)\right) \\
&= \int_{A}\{f(\omega)+g(\omega)\} \mathrm{d} \sigma\left(x^{*}, \mathscr{M}(\omega)\right)
\end{aligned}
$$

for each $A \in \mathscr{A}$ and $x^{*} \in \mathscr{X}^{*}$. Since $(\mathrm{KL}) \int_{A} f(\omega) \mathrm{d} \mathscr{M}(\omega),(\mathrm{KL}) \int_{A} g(\omega) \mathrm{d} \mathscr{M}(\omega) \in \mathscr{P}_{\text {wkc }}(\mathscr{X})$, we have $(\mathrm{KL}) \int_{A} f(\omega) \mathrm{d} \mathscr{M}(\omega)+(\mathrm{KL}) \int_{A} g(\omega) \mathrm{d} \mathscr{M}(\omega) \in \mathscr{P}_{\text {wkc }}(\mathscr{X})$. It follows that $f+g$ is $(\mathrm{KL}) \mathscr{M}$-integrable and

$$
\begin{aligned}
& (\mathrm{KL}) \int_{A}\{f(\omega)+g(\omega)\} \mathrm{d} \mathscr{M}(\omega) \\
& =(\mathrm{KL}) \int_{A} f(\omega) \mathrm{d} \mathscr{M}(\omega)+(\mathrm{KL}) \int_{A} g(\omega) \mathrm{d} \mathscr{M}(\omega)
\end{aligned}
$$

for each $A \in \mathscr{A}$. 
(2) In the same manner as in the proof of (1), we can show that $\lambda f$ is $\sigma\left(x^{*}, \mathscr{M}(\cdot)\right)$-integrable and

$$
\begin{aligned}
& \sigma\left(x^{*}, \lambda(\mathrm{KL}) \int_{A} f(\omega) \mathrm{d} \mathscr{M}(\omega)\right) \\
& =\lambda \sigma\left(x^{*},(\mathrm{KL}) \int_{A} f(\omega) \mathrm{d} \mathscr{M}(\omega)\right) \\
& =\lambda \int_{A} f(\omega) \mathrm{d} \sigma\left(x^{*}, \mathscr{M}(\omega)\right) \\
& =\int_{A} \lambda f(\omega) \mathrm{d} \sigma\left(x^{*}, \mathscr{M}(\omega)\right)
\end{aligned}
$$

for each $x^{*} \in \mathscr{X}^{*}, A \in \mathscr{A}$, and $\lambda \geq 0$. Since (KL) $\int_{A} f(\omega)$ $\mathrm{d} \mathscr{M}(\omega) \in \mathscr{P}_{\text {wkc }}(\mathscr{X})$, we have $\lambda(\mathrm{KL}) \int_{A} f(\omega) \mathrm{d} \mathscr{M}(\omega) \in$ $\mathscr{P}_{\text {wkc }}(\mathscr{X})$. Thus $\lambda f$ is $(\mathrm{KL}) \mathscr{M}$-integrable and

$$
(\mathrm{KL}) \int_{A} \lambda f(\omega) \mathrm{d} \mathscr{M}(\omega)=\lambda(\mathrm{KL}) \int_{A} f(\omega) \mathrm{d} \mathscr{M}(\omega)
$$

for each $A \in \mathscr{A}$. This completes the proof.

One of the most important properties of the KluvánekLewis integral is that the indefinite integral of an integrable function is a vector measure. We would like to extend the result to set-valued Kluvánek-Lewis integrals as follows.

Theorem 7. Let $(\Omega, \mathscr{A}, \mu)$ be a nonnegative finite measure space, $\mathscr{M}: \mathscr{A} \rightarrow \mathscr{P}_{w k c}(\mathscr{X})$ a $\mu$-continuous set-valued measure, and $f: \Omega \rightarrow \mathbb{R}(K L) \mathscr{M}$-integrable. Then $\mathscr{M}^{\prime}:$ $\mathscr{A} \rightarrow \mathscr{P}_{w k c}(\mathscr{X})$ defined by

$$
\mathscr{M}^{\prime}(A)=(K L) \int_{A} f(\omega) \mathrm{d} \mathscr{M}(\omega) \quad \forall A \in \mathscr{A}
$$

is a $\mu$-continuous set-valued measure.

Proof. Note that $\mathscr{M}^{\prime}: \mathscr{A} \rightarrow \mathscr{P}_{\text {wkc }}(\mathscr{X})$ is a set-valued measure if and only if $\sigma\left(x^{*}, \mathscr{M}^{\prime}(\cdot)\right)$ is a scalar-valued measure for each $x^{*} \in \mathscr{X}^{*}$. Thus, to end the proof, we show that $\sigma\left(x^{*}\right.$, $\left.\mathscr{M}^{\prime}(\cdot)\right)$ is a scalar-valued measure for each $x^{*} \in \mathscr{X}^{*}$. Obviously,

$$
\begin{aligned}
\sigma & \left(x^{*}, \mathscr{M}^{\prime}(\emptyset)\right) \\
& =\sigma\left(x^{*},(\mathrm{KL}) \int_{\emptyset} f(\omega) \mathrm{d} \mathscr{M}(\omega)\right) \\
& =\int_{\emptyset} f(\omega) \mathrm{d} \sigma\left(x^{*}, \mathscr{M}(\omega)\right)=0
\end{aligned}
$$

for each $x^{*} \in \mathscr{X}^{*}$. In the following, we prove countable additivity of $\sigma\left(x^{*}, \mathscr{M}^{\prime}(\cdot)\right)$. For each $x^{*} \in \mathscr{X}^{*}$ and $A \in \mathscr{A}$, we have

$$
\begin{aligned}
\sigma\left(x^{*}, \mathscr{M}^{\prime}(A)\right) & =\sigma\left(x^{*},(\mathrm{KL}) \int_{A} f(\omega) \mathrm{d} \mathscr{M}(\omega)\right) \\
& =\int_{A} f(\omega) \mathrm{d} \sigma\left(x^{*}, \mathscr{M}(\omega)\right) .
\end{aligned}
$$

Since $f$ is $\sigma\left(x^{*}, \mathscr{M}(\omega)\right)$-integrable, it is known from standard scalar-valued measure theory that $A \mapsto \int_{A} f(\omega) \mathrm{d} \sigma\left(x^{*}\right.$, $\mathscr{M}(\omega))$ is countably additive. Thus, if $\left\{A_{n}\right\}_{n \in \mathbb{N}}$ is a sequence of pairwise disjoint elements of $\mathscr{A}$, we have

$$
\begin{aligned}
\sigma\left(x^{*}, \mathscr{M}^{\prime}\left(\bigcup_{n=1}^{\infty} A_{n}\right)\right) & =\sigma\left(x^{*},(\mathrm{KL}) \int_{\cup_{n=1}^{\infty} A_{n}} f(\omega) \mathrm{d} \mathscr{M}(\omega)\right) \\
& =\int_{\cup_{n=1}^{\infty} A_{n}} f(\omega) \mathrm{d} \sigma\left(x^{*}, \mathscr{M}(\omega)\right) \\
& =\sum_{n=1}^{\infty} \int_{A_{n}} f(\omega) \mathrm{d} \sigma\left(x^{*}, \mathscr{M}(\omega)\right) \\
& =\sum_{n=1}^{\infty} \sigma\left(x^{*},(\mathrm{KL}) \int_{A_{n}} f(\omega) \mathrm{d} \mathscr{M}(\omega)\right) \\
& =\sum_{n=1}^{\infty} \sigma\left(x^{*}, \mathscr{M}^{\prime}\left(A_{n}\right)\right) ;
\end{aligned}
$$

that is, $\sigma\left(x^{*}, \mathscr{M}^{\prime}(\cdot)\right)$ is countably additive. So, we conclude that $\sigma\left(x^{*}, \mathscr{M}^{\prime}(\cdot)\right)$ is a scalar-valued measure for each $x^{*} \epsilon$ $\mathscr{X}^{*}$. It follows that $\mathscr{M}^{\prime}: \mathscr{A} \rightarrow \mathscr{P}_{\mathrm{wkc}}(\mathscr{X})$ is a set-valued measure.

By Theorem 6.3.2 [9], $\mathscr{M}$ is a $\mu$-continuous set-valued measure if and only if $\sigma\left(x^{*}, \mathscr{M}(\cdot)\right)$ is a $\mu$-continuous scalarvalued measure for each $x^{*} \in \mathscr{X}^{*}$. It follows that $\sigma\left(x^{*}, \mathscr{M}^{\prime}(\cdot)\right)$ is $\mu$-continuous for each $x^{*} \in \mathscr{X}^{*}$. Again by Theorem 6.3.2 [9], $\mathscr{M}^{\prime}$ is a $\mu$-continuous set-valued measure. This completes the proof.

Theorem 8. Suppose that $\mathscr{X}$ has the RNP, $\mathscr{X}^{*}$ is separable, $\mathscr{M}: \mathscr{A} \rightarrow \mathscr{P}_{w k c}(\mathscr{X})$ is a $\mu$-continuous set-valued measure of bounded variation, and $f: \Omega \rightarrow \mathbb{R}_{+}$is (KL) M-integrable. Then there exists an integrably bounded set-valued function $X: \Omega \rightarrow \mathscr{P}_{w k c}(\mathscr{X})$ such that

$$
(K L) \int_{\Omega} f(\omega) \mathrm{d} \mathscr{M}(\omega)=\int_{\Omega} f(\omega) X(\omega) \mathrm{d} \mu(\omega) .
$$

Proof. Since $\mathscr{X}$ has the RNP, $\mathscr{X}^{*}$ is separable and $\mathscr{M}: \mathscr{A} \rightarrow$ $\mathscr{P}_{\text {wkc }}(\mathscr{X})$ is a $\mu$-continuous set-valued measure of bounded variation, by Theorem 6.4.4 [9], there exists an integrably bounded set-valued function $X: \Omega \rightarrow \mathscr{P}_{\text {wkc }}(\mathscr{X})$ which is a Radon-Nikodým derivative of $\mathscr{M}$ with respect to $\mu$. Then we have

$$
\mathscr{M}(A)=\int_{A} X(\omega) \mathrm{d} \mu(\omega)
$$

for each $A \in \mathscr{A}$. It follows that

$$
\begin{aligned}
\sigma\left(x^{*}, \mathscr{M}(A)\right) & =\sigma\left(x^{*}, \int_{A} X(\omega) \mathrm{d} \mu(\omega)\right) \\
& =\int_{A} \sigma\left(x^{*}, X(\omega)\right) \mathrm{d} \mu(\omega)
\end{aligned}
$$

for each $x^{*} \in \mathscr{X}^{*}$ and each $A \in \mathscr{A}$. Note that $\mathscr{M}: \mathscr{A} \rightarrow$ $\mathscr{P}_{\text {wkc }}(\mathscr{X})$ is a $\mu$-continuous set-valued measure if and only 
if $\sigma\left(x^{*}, \mathscr{M}(\cdot)\right)$ is a $\mu$-continuous scalar-valued measure for each $x^{*} \in \mathscr{X}^{*}$, which, together with the above equality, implies that $\sigma\left(x^{*}, X(\omega)\right)$ is a Radon-Nikodým derivative of $\sigma\left(x^{*}, \mathscr{M}(\cdot)\right)$ with respect to $\mu$ for each $x^{*} \in \mathscr{X}^{*}$. Hence, we have

$$
\begin{aligned}
\sigma & \left(x^{*},(\mathrm{KL}) \int_{\Omega} f(\omega) \mathrm{d} \mathscr{M}(\omega)\right) \\
& =\int_{\Omega} f(\omega) \mathrm{d} \sigma\left(x^{*}, \mathscr{M}(\omega)\right) \\
& =\int_{\Omega} f(\omega) \sigma\left(x^{*}, X(\omega)\right) \mathrm{d} \mu(\omega)
\end{aligned}
$$

for each $x^{*} \in \mathscr{X}^{*}$. Since $f$ is nonnegative, by Proposition 3.4 [25], we have

$$
\begin{aligned}
& \int_{\Omega} f(\omega) \sigma\left(x^{*}, X(\omega)\right) \mathrm{d} \mu(\omega) \\
& =\int_{\Omega} \sigma\left(x^{*}, f(\omega) X(\omega)\right) \mathrm{d} \mu(\omega) \\
& =\sigma\left(x^{*}, \int_{\Omega} f(\omega) X(\omega) \mathrm{d} \mu(\omega)\right)
\end{aligned}
$$

for each $x^{*} \in \mathscr{X}^{*}$. This, together with equality (30), follows that

$$
(\mathrm{KL}) \int_{\Omega} f(\omega) \mathrm{d} \mathscr{M}(\omega)=\int_{\Omega} f(\omega) X(\omega) \mathrm{d} \mu(\omega) .
$$

This completes the proof.

Theorem 9. Let $\mathscr{M}: \mathscr{A} \rightarrow \mathscr{P}_{w k c}(\mathscr{X})$ be a set-valued measure and let $f: \Omega \rightarrow \mathbb{R}$ be $(K L) M$ M-integrable. Then

$$
\left|(K L) \int_{A} f(\omega) \mathrm{d} \mathscr{M}(\omega)\right| \leq \int_{A}|f(\omega)| \mathrm{d}(\omega)
$$

for each $A \in \mathscr{A}$.

Proof. Since $f$ is (KL) $\mathscr{M}$-integrable, there exists $(\mathrm{KL}) \int_{A} f(\omega) \mathrm{d} \mathscr{M}(\omega) \in \mathscr{P}_{\text {wkc }}(\mathscr{X})$ such that

$$
\sigma\left(x^{*},(\mathrm{KL}) \int_{A} f(\omega) \mathrm{d} \mathscr{M}(\omega)\right)=\int_{A} f(\omega) \mathrm{d} \sigma\left(x^{*}, \mathscr{M}(\omega)\right)
$$

for each $A \in \mathscr{A}$ and $x^{*} \in \mathscr{X}^{*}$. From the properties of support functions, we have

$$
\begin{aligned}
& \left|(\mathrm{KL}) \int_{A} f(\omega) \mathrm{d} \mathscr{M}(\omega)\right| \\
& =H\left((\mathrm{KL}) \int_{A} f(\omega) \mathrm{d} \mathscr{M}(\omega),\{0\}\right) \\
& =\sup _{\left\|x^{*}\right\| \leq 1}\left|\sigma\left(x^{*},(\mathrm{KL}) \int_{A} f(\omega) \mathrm{d} \mathscr{M}(\omega)\right)\right| \\
& =\sup _{\left\|x^{*}\right\| \leq 1}\left|\int_{A} f(\omega) \mathrm{d} \sigma\left(x^{*}, \mathscr{M}(\omega)\right)\right| \\
& \leq \sup _{\left\|x^{*}\right\| \leq 1} \int_{A}|f(\omega)| \mathrm{d}\left|\sigma\left(x^{*}, \mathscr{M}(\cdot)\right)\right|(\omega),
\end{aligned}
$$

where $\left|\sigma\left(x^{*}, \mathscr{M}(\cdot)\right)\right|$ is the total variation measure of $\sigma\left(x^{*}, \mathscr{M}(\cdot)\right)$. Note that $\left|\sigma\left(x^{*}, \mathscr{M}(\cdot)\right)\right|$ satisfies

$$
\begin{aligned}
\left|\sigma\left(x^{*}, \mathscr{M}(\cdot)\right)\right|(A) & =\sup \sum_{i=1}^{n}\left|\sigma\left(x^{*}, \mathscr{M}\left(A_{i}\right)\right)\right| \\
& \leq \sup \sum_{i=1}^{n}\left\|x^{*}\right\|\left|\mathscr{M}\left(A_{i}\right)\right| \\
& \leq \sup \sum_{i=1}^{n}\left|\mathscr{M}\left(A_{i}\right)\right| \\
& =|\mathscr{M}|(A)
\end{aligned}
$$

for each $A \in \mathscr{A}$ and $x^{*} \in \mathscr{X}^{*}$ with $\left\|x^{*}\right\| \leq 1$, where the supremum is taken over all finite measurable partitions $\left\{A_{1}, \ldots, A_{n}\right\}$ of $A$. Since $|f(\omega)|$ is nonnegative, we have

$$
\int_{A}|f(\omega)| \mathrm{d}\left|\sigma\left(x^{*}, \mathscr{M}(\cdot)\right)\right|(\omega) \leq \int_{A}|f(\omega)| \mathrm{d}|\mathscr{M}|(\omega)
$$

for each $A \in \mathscr{A}$ and $x^{*} \in \mathscr{X}^{*}$ with $\left\|x^{*}\right\| \leq 1$. It follows that

$$
\begin{aligned}
\left|(\mathrm{KL}) \int_{A} f(\omega) \mathrm{d} \mathscr{M}(\omega)\right| & \leq \sup _{\left\|x^{*}\right\| \leq 1} \int_{A}|f(\omega)| \mathrm{d}\left|\sigma\left(x^{*}, \mathscr{M}(\omega)\right)\right| \\
& \leq \int_{A}|f(\omega)| \mathrm{d}|\mathscr{M}|(\omega)
\end{aligned}
$$

for each $A \in \mathscr{A}$. This completes the proof.

Corollary 10. Let $\mathscr{M}: \mathscr{A} \rightarrow \mathscr{P}_{w k c}(\mathscr{X})$ be a set-valued measure and let $f: \Omega \rightarrow \mathbb{R}$ be (KL) $\mathscr{M}$-integrable. Then

(1) $\lim _{A \rightarrow \emptyset} H\left((K L) \int_{A} f(\omega) \mathrm{d} \mathscr{M}(\omega),\{0\}\right)=0$;

(2) if $A=\emptyset$, then $(K L) \int_{A} f(\omega) \mathrm{d} \mathscr{M}(\omega)=\{0\}$;

(3) if $f=0$, then $(K L) \int_{A} f(\omega) \mathrm{d} \mathscr{M}(\omega)=\{0\}$ for each $A \in$ $\mathscr{A}$.

Proof. (1) By Theorem 9, we have

$$
\begin{aligned}
H\left((\mathrm{KL}) \int_{A} f(\omega) \mathrm{d} \mathscr{M}(\omega),\{0\}\right) & =\left|(\mathrm{KL}) \int_{A} f(\omega) \mathrm{d} \mathscr{M}(\omega)\right| \\
& \leq \int_{A}|f(\omega)| \mathrm{d}|\mathscr{M}|(\omega),
\end{aligned}
$$

which implies

$$
\begin{gathered}
\lim _{A \rightarrow \emptyset} H\left((\mathrm{KL}) \int_{A} f(\omega) \mathrm{d} \mathscr{M}(\omega),\{0\}\right) \\
\leq \lim _{A \rightarrow \emptyset} \int_{A}|f(\omega)| \mathrm{d}|\mathscr{M}|(\omega)=0 .
\end{gathered}
$$

Thus, $\lim _{A \rightarrow \emptyset} H\left((\mathrm{KL}) \int_{A} f(\omega) \mathrm{d} \mathscr{M}(\omega),\{0\}\right)=0$. 
(2) If $A=\emptyset$, then $\int_{A}|f(\omega)| \mathrm{d}|\mathscr{M}|(\omega)=0$. This implies

$$
\begin{gathered}
H\left((\mathrm{KL}) \int_{A} f(\omega) \mathrm{d} \mathscr{M}(\omega),\{0\}\right) \\
\quad=\left|(\mathrm{KL}) \int_{A} f(\omega) \mathrm{d} \mathscr{M}(\omega)\right| \\
\quad \leq \int_{A}|f(\omega)| \mathrm{d}|\mathscr{M}|(\omega)=0 ;
\end{gathered}
$$

that is, $H\left((\mathrm{KL}) \int_{A} f(\omega) \mathrm{d} \mathscr{M}(\omega),\{0\}\right)=0$. Since $\left(\mathscr{P}_{\text {wkc }}(\mathscr{X}), H\right)$ is a complete metric space, we have $(\mathrm{KL}) \int_{A} f(\omega) \mathrm{d} \mathscr{M}(\omega)=$ $\{0\}$.

(3) If $f=0$, then

$$
\begin{aligned}
& \sigma\left(x^{*},(\mathrm{KL}) \int_{A} f(\omega) \mathrm{d} \mathscr{M}(\omega)\right) \\
& =\int_{A} f(\omega) \mathrm{d} \sigma\left(x^{*}, \mathscr{M}(\omega)\right)=0
\end{aligned}
$$

for each $x^{*} \in \mathscr{X}^{*}$ and $A \in \mathscr{A}$, which implies that (KL) $\int_{A} f(\omega) \mathrm{d} \mathscr{M}(\omega)=\{0\}$ for each $A \in \mathscr{A}$. This completes the proof.

In the following, we give the Vitali type convergence theorem for the set-valued Kluvánek-Lewis integral.

Theorem 11. Let $\mathscr{M}: \mathscr{A} \rightarrow \mathscr{P}_{w k c}(\mathscr{X})$ be a set-valued measure and let $f, f_{n}: \Omega \rightarrow \mathbb{R}, n \in \mathbb{N}$, be (KL) M-integrable such that $\left\{f_{n}\right\}_{n \in \mathbb{N}}$ is uniformly integrable with respect to $|\mathscr{M}|$ and $\lim _{n \rightarrow \infty} f_{n}=f|\mathscr{M}|$-a.e. Then

$$
\begin{aligned}
& \lim _{n \rightarrow \infty} H\left((K L) \int_{\Omega} f_{n}(\omega) \mathrm{d} \mathscr{M}(\omega),\right. \\
& \left.(K L) \int_{\Omega} f(\omega) \mathrm{d} \mathscr{M}(\omega)\right)=0 .
\end{aligned}
$$

Proof. Since $f, f_{n}: \Omega \rightarrow \mathbb{R}, n \in \mathbb{N}$, are (KL) $\mathscr{M}$-integrable, there exist (KL) $\int_{\Omega} f_{n}(\omega) \mathrm{d} \mathscr{M}(\omega)$, (KL) $\int_{\Omega} f(\omega) \mathrm{d} \mathscr{M}(\omega) \in$ $\mathscr{P}_{\text {wkc }}(\mathscr{X}), n \in \mathbb{N}$, such that

$$
\begin{gathered}
\sigma\left(x^{*},(\mathrm{KL}) \int_{\Omega} f_{n}(\omega) \mathrm{d} \mathscr{M}(\omega)\right) \\
=\int_{\Omega} f_{n}(\omega) \mathrm{d} \sigma\left(x^{*}, \mathscr{M}(\omega)\right) \\
\sigma\left(x^{*},(\mathrm{KL}) \int_{\Omega} f(\omega) \mathrm{d} \mathscr{M}(\omega)\right)=\int_{\Omega} f(\omega) \mathrm{d} \sigma\left(x^{*}, \mathscr{M}(\omega)\right)
\end{gathered}
$$

for each $x^{*} \in \mathscr{X}^{*}$ and $n \in \mathbb{N}$. Then, by the properties of support functions, we have

$$
\begin{gathered}
H\left((\mathrm{KL}) \int_{\Omega} f_{n}(\omega) \mathrm{d} \mathscr{M}(\omega),(\mathrm{KL}) \int_{\Omega} f(\omega) \mathrm{d} \mathscr{M}(\omega)\right) \\
=\sup _{\left\|x^{*}\right\| \leq 1} \mid \sigma\left(x^{*},(\mathrm{KL}) \int_{\Omega} f_{n}(\omega) \mathrm{d} \mathscr{M}(\omega)\right) \\
-\sigma\left(x^{*},(\mathrm{KL}) \int_{\Omega} f(\omega) \mathrm{d} \mathscr{M}(\omega)\right) \mid \\
=\sup _{\left\|x^{*}\right\| \leq 1} \mid \int_{\Omega} f_{n}(\omega) \mathrm{d} \sigma\left(x^{*}, \mathscr{M}(\omega)\right) \\
\quad-\int_{\Omega} f(\omega) \mathrm{d} \sigma\left(x^{*}, \mathscr{M}(\omega)\right) \mid \\
\leq \sup _{\left\|x^{*}\right\| \leq 1} \int_{\Omega}\left|f_{n}(\omega)-f(\omega)\right| \mathrm{d}\left|\sigma\left(x^{*}, \mathscr{M}(\cdot)\right)\right|(\omega) .
\end{gathered}
$$

In the same manner as in the proof of Theorem 9, we have

$$
\begin{gathered}
\sup _{\left\|x^{*}\right\| \leq 1} \int_{\Omega}\left|f_{n}(\omega)-f(\omega)\right| \mathrm{d}\left|\sigma\left(x^{*}, \mathscr{M}(\omega)\right)\right| \\
\quad \leq \int_{\Omega}\left|f_{n}(\omega)-f(\omega)\right| \mathrm{d}|\mathscr{M}|(\omega) .
\end{gathered}
$$

Then, by classical Vitali's convergence theorem, we can conclude that

$$
\begin{aligned}
& H\left((\mathrm{KL}) \int_{\Omega} f_{n}(\omega) \mathrm{d} \mathscr{M}(\omega),(\mathrm{KL}) \int_{\Omega} f(\omega) \mathrm{d} \mathscr{M}(\omega)\right) \\
& \quad \leq \int_{\Omega}\left|f_{n}(\omega)-f(\omega)\right| \mathrm{d}|\mathscr{M}|(\omega) \longrightarrow 0
\end{aligned}
$$

as $n \rightarrow \infty$. This completes the proof.

Similarly, we can obtain the Lebesgue type dominated convergence theorem for the set-valued Kluvánek-Lewis integral as follows.

Theorem 12. Let $\mathscr{M}: \mathscr{A} \rightarrow \mathscr{P}_{w k c}(\mathscr{X})$ be a set-valued measure and let $f, f_{n}: \Omega \rightarrow \mathbb{R}, n \in \mathbb{N}$, be (KL) M-integrable such that $\lim _{n \rightarrow \infty} f_{n}=f|\mathscr{M}|$-a.e. If there exists a nonnegative, $|\mathscr{M}|$-integrable function $g: \Omega \rightarrow \mathbb{R}$ such that $\left|f_{n}(\omega)\right| \leq g(\omega)$ for all $n \in \mathbb{N}$ and $\omega \in \Omega$, then

$$
\lim _{n \rightarrow \infty} H\left(\int_{\Omega} f_{n}(\omega) \mathrm{d} \mathscr{M}(\omega), \int_{\Omega} f(\omega) \mathrm{d} \mathscr{M}(\omega)\right)=0 .
$$

Proof. By Theorem 9 and classical Lebesgue dominated convergence theorem, we can obtain the result.

In the last part of this section, we discuss the relationship between set-valued Bartle integrals and set-valued KluvánekLewis integrals. For convenience, we recall from [13] the definition of the set-valued Bartle integral as follows. 
Definition 13. Let $\mathscr{M}: \mathscr{A} \rightarrow \mathscr{P}_{0}(\mathscr{X})$ be a set-valued measure of bounded variation and let $f: \Omega \rightarrow \mathbb{R}$ be an element of $L^{1}(\Omega, \mathbb{R},|\mathscr{M}|)$. One defines the integral of $f$ with respect to $\mathscr{M}$, which one calls the set-valued Bartle integral, as follows:

$$
\int_{\Omega} f(\omega) \mathrm{d} \mathscr{M}(\omega)=\left\{\int_{\Omega} f(\omega) \mathrm{d} m(\omega): m \in S_{\mathscr{M}}\right\} .
$$

The vector-valued integrals of the right-hand side are defined in the sense of Bartle et al. [14].

In order to distinguish the set-valued Bartle integral from the set-valued Kluvánek-Lewis integral, in the sequel we denote the set-valued Bartle integral by (B) $\int_{\Omega} f(\omega) \mathrm{d} \mathscr{M}(\omega)$.

Theorem 14. Suppose that $\mathscr{X}$ has the $R N P, \mathscr{X}^{*}$ is separable, $\mathscr{M}: \mathscr{A} \rightarrow \mathscr{P}_{w k c}(\mathscr{X})$ is a set-valued measure of bounded variation, and $f: \Omega \rightarrow \mathbb{R}$ is an element of $L^{1}(\Omega, \mathbb{R},|\mathscr{M}|)$. Then $f: \Omega \rightarrow \mathbb{R}$ is $(K L) M$-integrable and

$$
(K L) \int_{\Omega} f(\omega) \mathrm{d} \mathscr{M}(\omega)=(\mathrm{B}) \int_{\Omega} f(\omega) \mathrm{d} \mathscr{M}(\omega) .
$$

Proof. Since $\mathscr{X}$ has the RNP, $\mathscr{X}^{*}$ is separable and $\mathscr{M}: \mathscr{A} \rightarrow$ $\mathscr{P}_{\text {wkc }}(\mathscr{X})$ is a set-valued measure of bounded variation; $\mathscr{M}$ and $f$ satisfy all the conditions of Theorem 6.5.4 [9]. Then, by Corollary 6.5.2 of Theorem 6.5.4 [9], we have

$$
\sigma\left(x^{*},(\mathrm{~B}) \int_{\Omega} f(\omega) \mathrm{d} \mathscr{M}(\omega)\right)=\int_{\Omega} f(\omega) \mathrm{d} \sigma\left(x^{*}, \mathscr{M}(\omega)\right)
$$

for each $x^{*} \in \mathscr{X}^{*}$ and, by Corollary 6.5.1 of Theorem 6.5.4 [9], we have

$$
\text { (B) } \int_{\Omega} f(\omega) \mathrm{d} \mathscr{M}(\omega) \in \mathscr{P}_{\mathrm{wkc}}(\mathscr{X}) \text {. }
$$

It follows that $f: \Omega \rightarrow \mathbb{R}$ is (KL) $\mathscr{M}$-integrable and

$$
(\mathrm{KL}) \int_{\Omega} f(\omega) \mathrm{d} \mathscr{M}(\omega)=(\mathrm{B}) \int_{\Omega} f(\omega) \mathrm{d} \mathscr{M}(\omega) .
$$

This completes the proof.

Remark 15. In the theory of vector-valued measures and integrals, Lewis ([23], Theorem 2.4) proves, using Vitali's convergence theorem, that Kluvánek-Lewis integral is equivalent to the one given by Bartle et al. [14]. In that case, the setvalued Bartle integral coincides with the set of KluvánekLewis integrals of $f$ with respect to measure selections of $\mathscr{M}$; that is,

$$
\text { (B) } \begin{aligned}
& \int_{\Omega} f(\omega) \mathrm{d} \mathscr{M}(\omega) \\
= & \left\{(\mathrm{KL}) \int_{\Omega} f(\omega) \mathrm{d} m(\omega): m \in S_{\mathscr{M}}\right\} .
\end{aligned}
$$

Thus, under the assumptions of Theorem 14, we can obtain that

$$
\begin{aligned}
(\mathrm{KL}) & \int_{\Omega} f(\omega) \mathrm{d} \mathscr{M}(\omega) \\
= & \left\{(\mathrm{KL}) \int_{\Omega} f(\omega) \mathrm{d} m(\omega): m \in S_{\mathscr{M}}\right\} .
\end{aligned}
$$

\section{Conclusion}

In the current paper we introduce a new integral of scalarvalued functions with respect to a set-valued measure. We discuss its properties and establish convergence theorems. In all applications which involve measure and integral, when measurement or data are set-valued, the structure defined in this paper can be applied. There are several directions for further investigation connected with this topic: specific properties of the integral, application on random case, expectation and conditional expectation, and application in economy.

\section{Conflict of Interests}

The authors declare that there is no conflict of interests regarding the publication of this paper.

\section{Acknowledgment}

The project is supported by the National Natural Science Foundation of China (11371002, 41201327), Specialized Research Fund for the Doctoral Program of Higher Education (20131101110048), Natural Science Foundation of Hebei Province (A2013201119), and Science and Technology Bureau of Baoding City (14ZF058).

\section{References}

[1] K. Vind, "Edgeworth allocations in an exchange economy with many traders," International Economic Review, vol. 5, pp. 165177, 1964.

[2] Z. Artstein, "Set-valued measures," Transactions of the American Mathematical Society, vol. 165, pp. 103-125, 1972.

[3] B. Cascales, V. Kadets, and J. Rodríguez, "Measurable selectors and set-valued Pettis integral in non-separable Banach spaces," Journal of Functional Analysis, vol. 256, no. 3, pp. 673-699, 2009.

[4] B. Cascales, V. Kadets, and J. Rodríguez, "Radon-Nikodým theorems for multimeasures in non-separable spaces," Journal of Mathematical Physics, Analysis, Geometry, vol. 9, no. 1, pp. 724, 2013.

[5] N. S. Papageorgiou, "On the theory of Banach space valued multifunctions. 2. Set valued martingales and set valued measures," Journal of Multivariate Analysis, vol. 17, no. 2, pp. 207-227, 1985.

[6] N. S. Papageorgiou, "Contributions to the theory of set valued functions and set valued measures," Transactions of the American Mathematical Society, vol. 304, no. 1, pp. 245-265, 1987.

[7] A. Precupanu and A. Gavriluţ, "A set-valued Egoroff type theorem," Fuzzy Sets and Systems, vol. 175, pp. 87-95, 2011.

[8] M. Stojakovic, "Set valued probability and its connection with set valued measure," Statistics \& Probability Letters, vol. 82, no. 6, pp. 1043-1048, 2012.

[9] W. Zhang, S. Li, Z. Wang, and Y. Gao, Set Valued Stochastic Process, Academic Press, Beijing, China, 2007.

[10] N. Dinculeanu, Vector Measures, vol. 95 of International Series of Monographs in Pure and Applied Mathematics, Pergamon Press, New York, NY, USA, 1967.

[11] D. A. Kandilakis, "On the extension of multimeasures and integration with respect to a multimeasure," Proceedings of the American Mathematical Society, vol. 116, no. 1, pp. 85-92, 1992. 
[12] I. Kluvànek and G. Knowles, Vector Measures and Control Systems, North-Holland, Amsterdam, The Netherlands, 1975.

[13] W.-Z. Wu, W.-X. Zhang, and R.-M. Wang, "Set valued Bartle integrals," Journal of Mathematical Analysis and Applications, vol. 255, no. 1, pp. 1-20, 2001.

[14] R. G. Bartle, N. Dunford, and J. Schwartz, "Weak compactness and vector measures," Canadian Journal of Mathematics, vol. 7, pp. 289-305, 1955.

[15] A. M. Precupanu and A. Croitoru, "A Gould type integral with respect to a multimeasure I," Analele Universitatii "Alexandru Ioan Cuza" Iasi, vol. 1, pp. 165-200, 2002.

[16] A. M. Precupanu and A. Croitoru, "A Gould type integral with respect to a multimeasure II," Analele Universitatii "Al. I. Cuza" Iasi, vol. 49, pp. 183-207, 2003.

[17] A. Gavriluţ, "On some properties of the Gould type integral with respect to a multisubmeasure," Analele Ştiinţifice ale Universităţii Al. I. Cuza din Iaşi, vol. 52, pp. 177-194, 2006.

[18] A. C. Gavriluţ, "A Gould type integral with respect to a multisubmeasure," Mathematica Slovaca, vol. 58, no. 1, pp. 4362, 2008.

[19] A. M. Precupanu and B. Satco, "The Aumann-Gould integral," Mediterranean Journal of Mathematics, vol. 5, no. 4, pp. 429-441, 2008.

[20] G. G. Gould, "Integration over vector-valued measures," Proceedings of the London Mathematical Society, vol. 15, pp. 193-225, 1965.

[21] A. Precupanu, A. Gavriluț, and A. Croitoru, "A fuzzy Gould type integral," Fuzzy Sets and Systems, vol. 161, no. 5, pp. 661-680, 2010.

[22] I. Kluvànek, "Fourier transforms of vector-valued functions and measures," Studia Mathematica, vol. 37, pp. 1-12, 1970.

[23] D. R. Lewis, "Integration with respect to vector measures," Pacific Journal of Mathematics, vol. 33, pp. 157-165, 1970.

[24] T. V. Panchapagesan, The Bartle-Dunford-Schwartz Integral: Integration with Respect to a Sigma-Additive Vector Measure, Birkhäuser, Basel, Switzerland, 2008.

[25] N. S. Papageorgiou, "On the theory of Banach space valued multifunctions. I. Integration and conditional expectation," Journal of Multivariate Analysis, vol. 17, no. 2, pp. 185-206, 1985. 


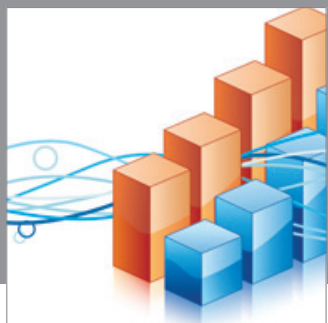

Advances in

Operations Research

mansans

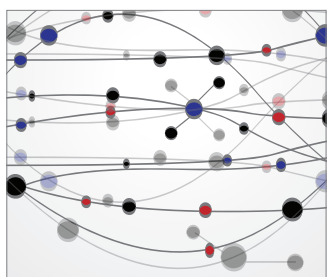

The Scientific World Journal
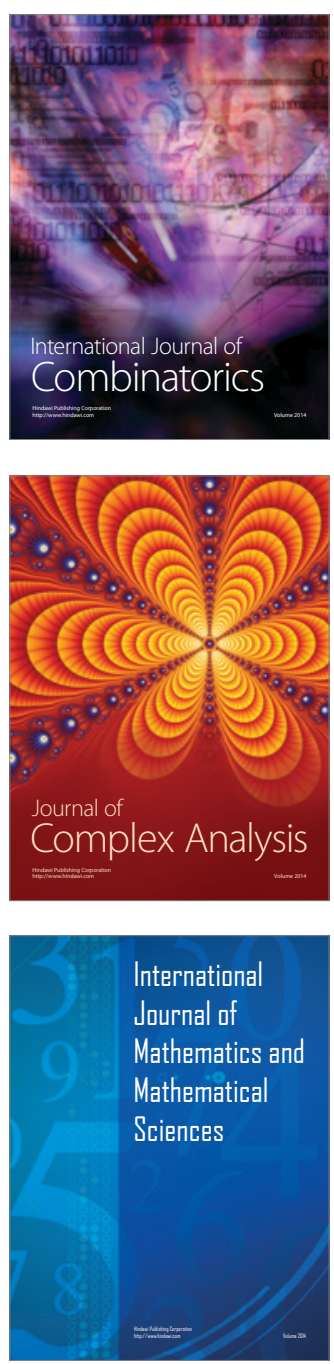
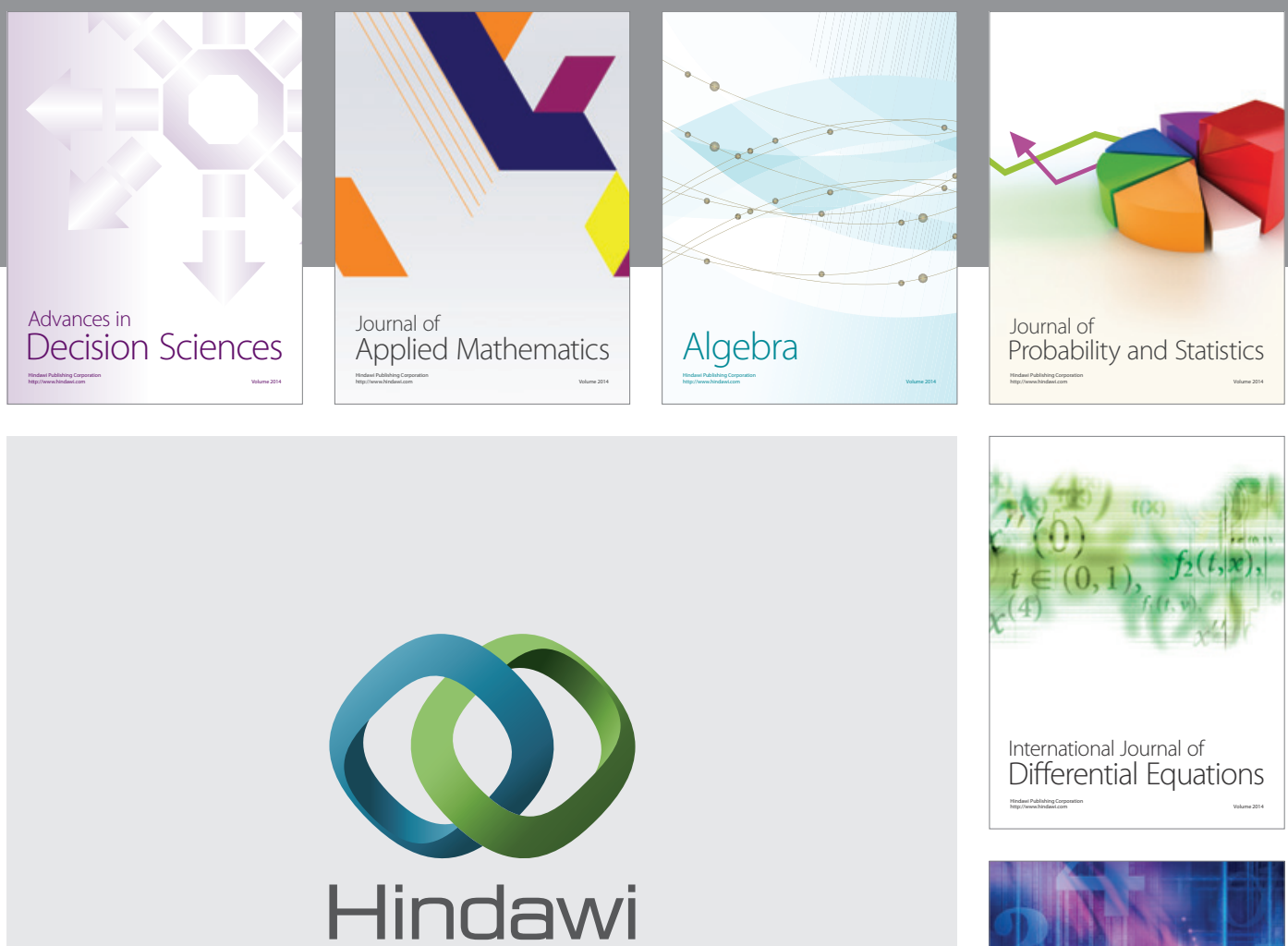

Submit your manuscripts at http://www.hindawi.com
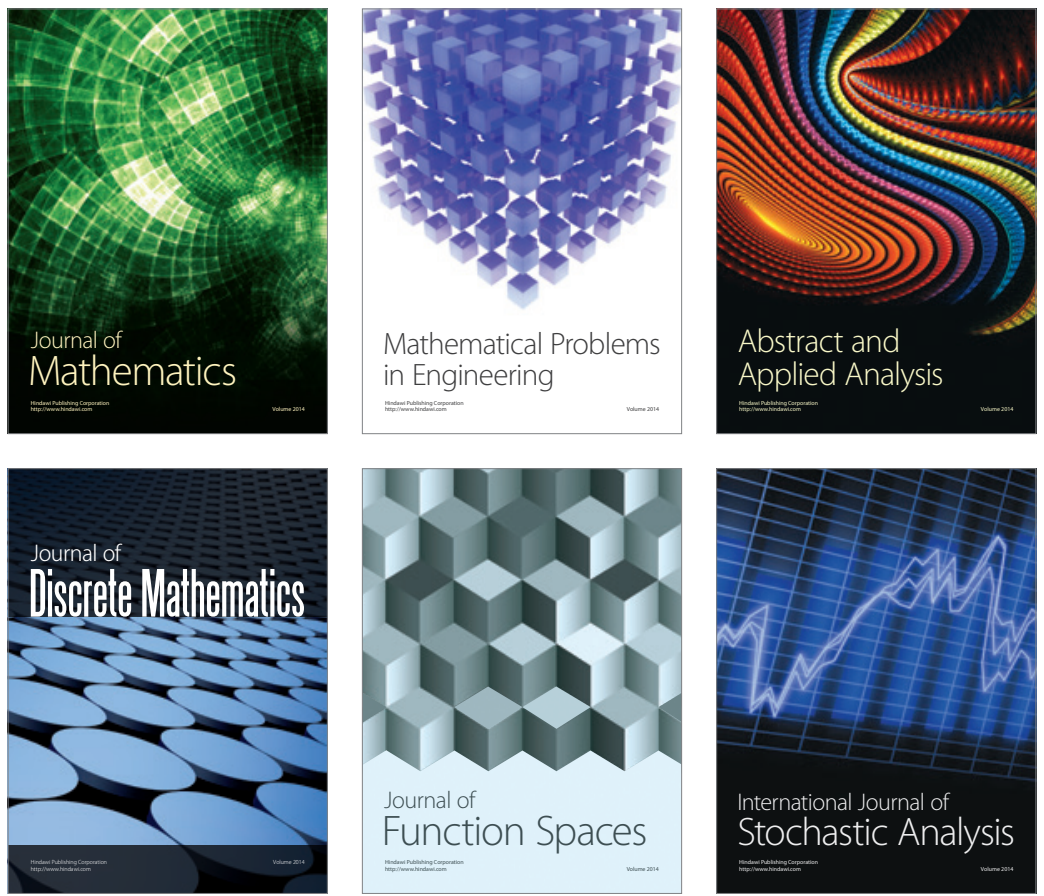

Journal of

Function Spaces

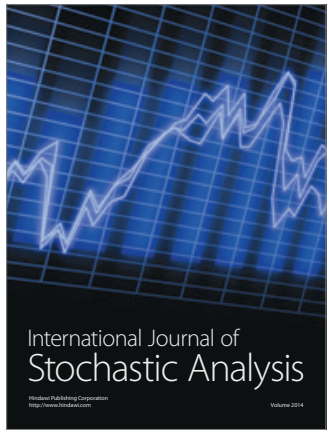

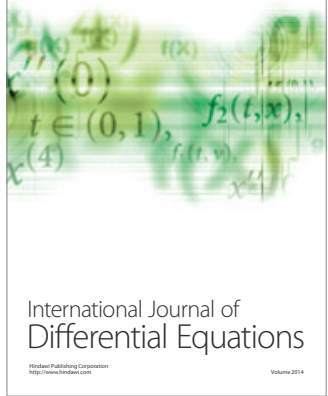
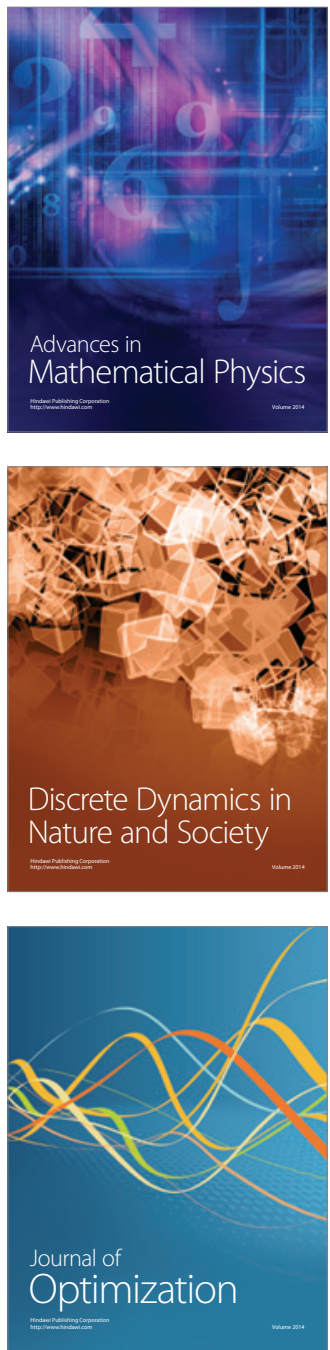